\title{
Increasing efficiency for dried blood spot analysis: prospects for automation and simplified sample analysis
}

"There is an emerging need for greater efficiency, either through automation of the sample preparation process or alternative approaches that require minimal or no sample preparation, such as direct analysis of dried blood spots."

Keywords: automation $\approx$ DART $\approx$ DESI $₫$ direct desorption $\approx$ direct elution $\approx$ dried blood spot

Dried blood spot (DBS) sampling [1-3] is the collection of blood on an absorbent storage medium prior to analysis of the sample. Reported use of DBS sampling dates back to 1963 when it was first used for the screening of neonatal metabolic disorders [4]. Recently, there has been increasing interest in the use of DBS to support pharmacokinetic and toxicokinetic studies in small-molecule drug discovery and development. The key features of DBS samples are the low blood volume requirement, potentially leading to decreased animal use, and the potential for simplified sample collection, storage and shipment conditions. Although there is interest in the use of DBS for large molecules, the majority of reports currently in the literature are focused on small molecules. Hence, the focus of this article is on the use of DBS in small-molecule bioanalysis.

The standard sample preparation approach for DBS analysis consists of punching out a disk from the card that contains the DBS followed by extraction of the analyte. The punched sample disks typically range in diameter from 3 to $8 \mathrm{~mm}$ and are extracted with an organic solvent, or a mixture of aqueous and organic solvent. Internal standard (IS) is usually added to the extraction solvent. The extract is then analyzed by LC-MS/MS. This standard approach (extraction of analytes providing a liquid sample for LC-MS/MS analysis) is likely to be suitable for the vast majority of compounds. However, performing this process manually is time consuming and labor intensive. There is an emerging need for greater efficiency, either through automation of the sample preparation process or alternative approaches that require minimal or no sample preparation, such as direct analysis of DBS.

\section{Automation for DBS analysis}

Automation of DBS analysis usually involves the robotic punching of DBS cards followed by adding solvent for extraction. The process can be fully or semi-automated. A barcode reader can be added for sample identification to streamline the automation process. In a semi-automated system, sample cards are fed in manually, while in a fully automated system, the cards are fed by grippers and, thus, require no operator attention. A disk detection system ensures that the punched disk is placed in the correct well of the 96- or 384well collection plate. In a fully automated system, a robotic arm can pick up the sample plates and place them onto a liquid-handling workstation for the downstream processing. There are numerous fully and semi-automated card punching systems commercially available. These include instruments from BSD Robotics [101], PerkinElmer [102], Hudson Robotics [103] and other manufacturers. Some of the automation systems can accommodate different card formats. For example, the BSD-1000 can handle card sizes of $50 \times 50,85 \times 50$ and $108 \times 50 \mathrm{~mm}$. The options for automation systems are likely to increase as DBS collection becomes more widely adopted for bioanalysis.

\section{Direct elution of DBS samples}

The DBS samples can also be analyzed without prior extraction using either direct elution [5-9] or direct desorption [10,11] techniques followed by MS detection. The use of direct online elution of analytes from DBS samples was first reported by Deglon et al. [5]. However, this approach required the prepunching of DBS samples and the mounting of each punched disk in an online holder. In addition, this system had limited

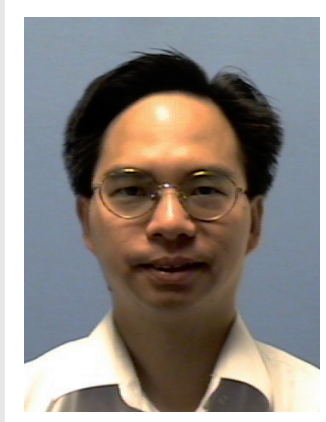

Philip Wong

Author for correspondence:

Department of Pharmacokinetics \&

Drug Metabolism, One Amgen

Center Drive, Thousand Oaks,

CA 91320-1799, USA

Tel.: +l 8053134701

Fax: + I 8054994868

E-mail: pwong@amgen.com

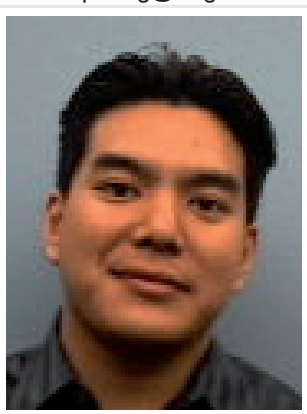

Roger Pham

Department of Pharmacokinetics \& Drug Metabolism, One Amgen

Center Drive, CA 91320-1799, USA

Authors continued overleaf... 


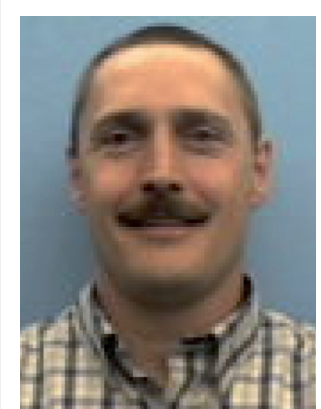

Bernd A Bruenner

Department of Pharmacokinetics \& Drug Metabolism, One Amgen Center Drive, CA 91320-1799, USA

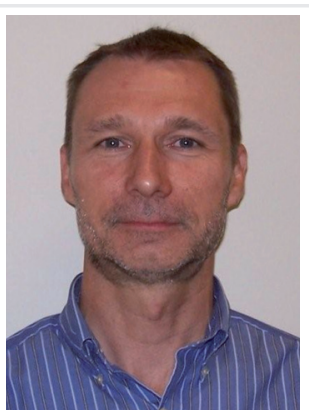

Christopher A James Department of Pharmacokinetics \& Drug Metabolism, One Amgen Center Drive, CA 91320-1799, USA sample capacity and is not suitable for highthroughput analysis. An alternative approach for direct elution is the use of a TLC-MS interface $[6,7]$ or liquid-extraction surface sampling coupled to chip-based robotic nanoelectrospray MS [8,9]. The TLC-MS interface was designed to extract compounds from a TLC plate and does not require punching of DBS samples. Samples are eluted online directly into a LC-MS/MS system. Currently, this system can only handle a limited number of samples (four DBS cards) at one time and needs further development for automated high-throughput bioanalysis.

\section{"...improvements in efficiency are required to fully exploit the benefits of dried blood spots."}

A system using liquid extraction surface sampling coupled to chip-based robotic nanoelectrospray MS is marketed by Advion as TriVersa ${ }^{\mathrm{TM}}-\mathrm{Nanomate}^{\circledR}$. The TriVersaNanomate system requires manual punching and mounting of the DBS samples on a plate using double-sided tape [8]. The key step is the formation of a liquid microjunction by allowing the extraction solvent from the sampling end of a tip to extend out and touch the DBS surface where extraction takes place. The extraction solvent is then drawn back into the tip and moves to the mass spectrometer interface where a high voltage is applied to the tip and nanoelectrospray ionization is initiated. The tip is disposed after each sample analysis so there is no carryover. Recently, the system has been modified to allow the direct analysis of DBS samples on a card [9] and does not require punching. The analysis time for each sample is usually less than $1 \mathrm{~min}$. However, the TriVersa-Nanomate system suffers from poor sensitivity due to matrix ion suppression. In addition, due to the absence of chromatographic separation, the analysis may have interference from certain metabolites (e.g. $\mathrm{N}$-oxide and direct glucuronide) that can decompose back to the parent compound in the ionization source of the mass spectrometer.

\section{Direct desorption of DBS samples}

Direct desorption of DBS samples can be achieved by ambient-ionization MS techniques $[12,13]$ such as desorption electrospray ionization (DESI) [10] and direct analysis in real time $\left(\mathrm{DART}^{\circledR}\right)$ [11]. DESI was developed by Cooks et al. in 2004 and is marketed commercially by Prosolia [14]. DART was developed in 2005 by Cody and Laramee and is marketed commercially by IonSense [15]. For analysis by DESI, DBS cards are cut into strips and secured onto a microscope glass slide whereas blood is spotted directly on a glass slide for analysis by DART. The surface is moved in one direction at constant velocity so that an ion spray in a DESI source or metastable gas phase species (helium) in a DART source desorbs analytes directly from the surface of the DBS without prior sample preparation. These direct desorption techniques greatly increase the throughput of DBS sample analyses by eliminating the extraction step and by utilizing short analysis times (from seconds to a minute). However, they also suffer from the absence of chromatography resulting in poor sensitivity due to ion suppression and possible interference from decomposition of metabolites.

\section{Future perspective}

Analysis with DBS has unique features that can markedly reduce blood sample volumes and simplify sampling logistics for both preclinical and clinical studies. However, current approaches are not optimal for processing the large numbers of samples generated in pharmacokinetic studies, and improvements in efficiency are required to fully exploit the benefits of DBS. An improvement required is the automation of manual sample-handling steps so that extracted analytes can be analyzed by standard analytical methods such as LC-MS/MS. These methods have the advantage of being familiar to scientists and widely accepted by regulatory authorities. Consideration should not, however, be limited to established techniques when novel approaches can remove multiple steps in the analysis. Methods involving direct elution or direct desorption should be acceptable for regulatory purposes if the appropriate validation specifications can be met. However, current direct analysis approaches still need significant improvement to match the wide applicability and performance of LC-MS/MS. Other aspects of DBS could also potentially be improved. Examples include DBS card materials that improve analyte extractability or ionization efficiency with direct desorption techniques, or cards and holders designed specifically for efficient automation, automatic blood spot recognition, and card identification and transport in the instrument. Introduction of an IS into the solvent used to extract the DBS is potentially inferior to current bioanalytical approaches. Possible alternatives could include spraying of IS onto the spot or impregnating IS into the card. Automated blood sample collection using DBS could also be a useful development [16]. Current automated blood collection 
systems for preclinical studies involve the collection and storage of blood in a refrigerated fraction collector [17]. Direct spotting of blood onto DBS cards by an automated blood sampling system would have the dual advantages of automating spotting on the cards whilst exploiting the inherent stability of many analytes in the DBS format at ambient temperature.

\section{Bibliography}

1 Li W, Tse FLS. Dried blood spot sampling in combination with LC-MS/MS for quantitative analysis of small molecules. Biomed. Chromatogr. 24, 49-65 (2010).

2 Patel P, Mulla H, Tanna S, Pandya H. Facilitating pharmacokinetic studies in children: a new use of dried blood spots. Arch. Dis. Child. 95, 484-487 (2010).

3 Spooner N. A glowing future for dried blood spot sampling. Bioanalysis 2, 1343-1344 (2010).

4 Guthrie R, Susi A. A simple phenylalanine method for detecting phenylketonuria in large populations of newborn infants. Pediatrics 32, 338-343 (1963).

5 Deglon J, Thomas A, Cataldo A, Mangin P, Staub C. On-line desorption of dried blood spot: a novel approach for the direct LC/MS analysis of $\mu$-whole blood samples. J. Pharm. Biomed. Anal. 49, 1034-1039 (2009).

6 Abu-Rabie P, Spooner N. Direct quantitative bioanalysis of drugs in dried blood spot samples using a thin-layer chromatography mass spectrometer interface. Anal. Chem. 81, 10275-10284 (2009).

7 Abu-Rabie P, Spooner N, Loppacher M. Direct quantitative bioanalysis of drugs in dried blood spot samples. Presented at: $58^{\text {th }}$ ASMS Conference on Mass Spectrometry and Allied Topics, Salt Lake City, UT, USA, 23-27 May, 2010.

\section{Financial \& competing interests disclosure}

The authors are employed by Amgen Inc. The authors have no other relevant affiliations or financial involvement with any organization or entity with a financial interest in or financial conflict with the subject matter or materials discussed in the manuscript apart from those disclosed.

No writing assistance was utilized in the production of this manuscript.

8 Kertesz V, Van Berkel GJ. Fully automated liquid extraction-based surface sampling and ionization using a chip-based robotic nanoelectrospray platform. J. Mass Spectrom. 45, 252-260 (2010).

9 Henion J, Eikel D, Rule G, Vega J, Prosser S, Jones J. Liquid extraction surface analysis (LESA) of dried blood spot cards via chip-based nanoelectrospray for drug and drug metabolite monitoring studies. Presented at: $58^{\text {th }}$ ASMS Conference on Mass Spectrometry and Allied Topics. Salt Lake City, UT, USA, 23-27 May, 2010.

10 Wiseman JM, Evans CA, Bowen CL, Kennedy JH. Direct analysis of dried blood spots utilizing desorption electrospray ionization (DESI) mass spectrometry. Analyst 135, 720-725 (2010).

11 Gordon J, Crawford E, Wu JT, Musselman BD et al. Quantitative analysis of dried blood spots by DART (direct analysis in real time)/MS/MS without sample preparation. Presented at: $58^{t h}$ ASMS Conference on Mass Spectrometry and Allied Topics. Salt Lake City, UT, USA, 23-27 May, 2010.

12 Weston DJ. Ambient ionization mass spectrometry: current understanding of mechanistic theory; analytical performance and application areas. Analyst 136, 661-668 (2010).

13 Cooks RG, Ouyang Z, Takats Z, Wiseman JM. Ambient mass spectrometry. Science 311, 1566-1570 (2006).
14 Takats Z, Wiseman JM, Gologan B, Cooks RG. Mass-spectrometry sampling under ambient conditions with desorption electrospray ionization. Science 306, 471-473 (2004).

15 Cody RB, Laramee RB. Versatile new ion source for analysis of materials in open air under ambient conditions. Anal Chem. 77, 2297-2302 (2005).

16 Pham R, Wong P, Soto M et al. Application of automated serial blood sampling in mice and dried blood spot technique using LC-MS/MS for pharmacokinetic studies. Presented at $58^{\text {th }}$ ASMS Conference on Mass Spectrometry and Allied Topics. Salt Lake City, UT, USA, 23-27 May, 2010.

17 Gunaratna PC, Kissinger PT, Kissinger CB, Gitzen JF. An automated blood sampler for simultaneous sampling of systemic blood and brain microdialysates for drug absorption, distribution, metabolism and elimination studies. J. Pharmacol. Toxicol. 49, 57-64 (2004).

\section{- Websites}

101 BSD Robotics. www.bsdrobotics.com/main.htm

102 PerkinElmer. www.perkinelmer.com

103 Hudson Robotics. www.hudsoncontrol.com 